\title{
ON A SET OF INTERDEPARTURE TIME DISTRIBUTIONS OF THE $M / G / 1$ QUEUE WITH SERVER VACATIONS
}

\author{
Shinsuke Shimogawa Yoshitaka Takahashi \\ NTT Telecommunication Networks Laboratories
}

(Received June 24, 1991; Final May 17, 1993)

\begin{abstract}
This paper studies interdeparture time distributions of an $M / G / 1$ queue with server vacations provided a class of schedules for vacations. Parametric and ordering properties of the distributions and its perturbations are studied concerning the adopted vacation schedule and the length of vacations. We also discuss the results in view of the performance evaluation of a token-passing LAN.
\end{abstract}

\section{Introduction}

In a packet-oriented network, the departure processes of queues sometimes directly characterize the network performance. This is because the network congestion influences the communication quality through the arrival process of a packet stream at the destination terminal and because the arrival to the destination is sometimes directly given by the departure process of a queue. Such an example can be seen in token-passing LANs [1] as described below. In view of studying how transmission schedules affect the arrival process to the destination terminal in the LAN, this paper studies interdeparture time distributions of $M / G / 1$ queues with vacations [4].

First we note how the arrival process at the destination characterizes the communication quality. In an individual communication between terminals, the quality is determined by the number of packets which arrive within a fixed time. For example, in case of data transfers between computers, if the number of the packets is less than a threshold then the destination terminal requires a retransmission of packets and the transfer slows down. In the cases of digitized voice transfers between telephones, each break of the threshold induces non-negligible noise. Thus, the less variability of the arrival process implies the better communication quality.

Let us describe how the departure process of a vacation model arises with the LANs. Consider a data transfer between terminals with text files, graphics, or voice messages in a lightly loaded token-passing LAN with a moderate speed. The arrival process at the destination terminal coincides with the departure process of the queue placed at the source. Therefore, the arrival process to the destination can be modeled by the departure process of an individual class of customers in polling models [13]. At the same time, we may often assume that such large amounts of data are not transported simultaneously. Thus, the packet stream for this data transfer can be assumed to perform the most of the traffic flow in the LAN, whereas the stream is loaded at the source terminal. Consequently, the arrival process to the destination may be modeled by the departure process of a vacation model with short vacation times.

We here consider the communication quality of the LAN concerning the adopted transmission schedule through the departure process of a vacation model, in which the transmission schedule corresponds to the schedule for vacations. We assume that the vacation model 
is an $M / G / 1$ type. As far as $M / G / 1$ type queues with schedules are concerned, the previous works seem to consider departure processes under a fixed schedule. Nain [9] derives the interdeparture time for individual classes of customers for $M / G / 1$ priority queue under the preemptive-resume service schedule, whereas Stanford [11] derives the distributions under the non-preemptive service schedule. Because there are various transmission schedules, we consider the set $\mathcal{T}$ of interdeparture time distributions given by changing the schedule for vacations, and then study the parametric and ordering properties of $\mathcal{T}$ and its perturbation $\left\{\mathcal{T}^{\varepsilon}\right\}$ near the ordinary queue. The parametric property will describe how changes of schedule can vary the interdeparture time distribution in the set of all probability distributions on non-negative real numbers, whereas the ordering property will describe how the variability of the interdeparture time depends on the schedules and on the length of vacations. These results may be qualitatively applied to the variability of the number of packets in a time interval and may give guiding information for studying LAN performances. This is because the interdeparture time distribution is one of the most natural one-dimensional characteristics of the departure processes. Disadvantages of this approach will be mentioned in Section 5.

This paper is organized as follows. Section 2 describes the vacation model. A class of vacation schedules is fixed. Section 3 presents the parametric property of the set $\mathcal{T}$. We show that $\mathcal{T}$ is given by a segment (1-simplex) in the space of probability distributions on nonnegative real numbers. Section 4 considers the convex ordering on $\mathcal{T}$ and on the perturbed

sets $\bigcup_{\varepsilon \text { near } 0} \mathcal{T}^{\varepsilon}$. The set $\mathcal{T}$ with convex order is shown to be identical to the interval [0,1] with the standard order. This result implies that the change of a vacation schedule which increases the number of customers in the system reduces the variability of the interdeparture times. We also study the increasing convex ordering along the perturbation $\left\{\mathcal{T}^{\varepsilon}\right\}_{\varepsilon \geq 0}$ near an ordinary $M / G / 1$ queue. We show that when vacation times are short, the variability of the interdeparture time decreases if the vacation time increases, except for such case as the exhaustive schedule. Section 5 interprets the results to discuss the performance of token-passing LANs.

\section{A queue with server vacations}

We describe here a vacation model refining the definition given in [4]. A sever of a queue is said to be on vacation when it stops serving, regardless of whether there are customers waiting in the queue or not. Consider an $M / G / 1 \infty$ queue with server vacations. We call the time when a vacation starts a vacation epoch. The schedule by which vacation epochs are determined is called a vacation schedule, so as not to confuse it with schedules for service order, which we call service schedules, such as FIFO and LIFO. The length of a vacation is called vacation time. The vacation time sequence is assumed to be i.i.d.. The arrival process, the service time sequence, and the vacation time sequence are assumed to be mutually independent. Let us specify classes of vacation and service schedules for which we study the interdeparture times of customers:

\section{Assumption 2.1.}

(i) The vacation schedule is assumed to be non-preemptive and multiple in the following sense:

Non-preemptive. A vacation can only start just after the completion of a service or a vacation.

Multiple. A new vacation starts if there are no customers waiting just after the 
completion of a service or a vacation.

(ii) The service schedule is also assumed to be non-preemptive.

(iii) The available information for vacation and service schedules, at time $t$, is contained in the histories of

- the queue length up to $t$,

- the arrival times of customers up to $t$,

- the lengths of the service and the vacations which started before $t$,

- processes which are independent of the arrival process, the vacation time sequence, and the service time sequence.

(iv) At least one customer is served if there are customers waiting just after the end of a vacation.

Note that assumption (iii) requires that the service and the vacation schedules do not refer to the service times of the waiting customers. Let $\mathcal{V}$ denote the set of all vacation schedules satisfying (i) through (iv) in Assumption 2.1. The probabilistic structure of the departure process is determined by quadruplet $\left(\lambda, \mu_{H}, \mu_{V}, \xi\right)$ where

$\lambda:$ the arrival rate,

$\mu_{H}:$ the service time distribution defined on $(0,+\infty)$,

$\mu_{V}:$ the vacation time distribution defined on $(0,+\infty)$,

$\xi$ : the vacation schedule contained in $\mathcal{V}$.

The following notation will be used:

$\mathbf{R}_{+}$: the set of all non-negative real numbers,

$h$ : the mean service time,

$v^{(j)}$ : the $j$-th moment of vacation time,

$v:=v^{(1)}$ (the mean vacation time),

$\rho:=\lambda h$ (traffic intensity),

$\sigma:=\int_{\mathbf{R}_{+}} e^{-\lambda x} \mu_{V}(d x)$,

The parameters $\lambda, h, v^{(j)}(j=1,2, \cdots)$ are assumed to be strictly positive and finite. Moreover, the quadruplet $\left(\lambda, \mu_{H}, \mu_{V}, \xi\right)$ is assumed that the queueing behavior of this model is stable in the ordinary sense that the departure rate is the same as the arrival rate. A sufficient stability condition under which the queue-length processes with vacation schedules $\xi \in \mathcal{V}$ are simultaneously stable can be given by

$$
\frac{\lambda v}{1-\rho}<1 \text { and } \rho<1
$$

This sufficiency condition can be shown by combining the well-known stability result for the 1-limited vacation schedule (e.g., [8]) and the sample-path comparison of the queue-length processes such as in [14].

\section{A parametric property of the distribution set $\mathcal{T}$}

Consider a triplet $\left(\lambda, \mu_{H}, \mu_{V}\right)$ satisfying the simultaneous stability condition (2.1) and fix this triplet. Let $\mu_{T}$ be the interdeparture time distribution in the steady state. We denote by $Q[\xi]$ the value of a quantity $Q$ under schedule $\xi \in \mathcal{V}$. This paper is mainly concerned with the following set of interdeparture time distributions:

$$
\mathcal{T}=\left\{\mu_{T}[\xi] \mid \xi \in v\right\}
$$

From here on, we use the notation

$\delta_{0}$ : the Dirac measure supported on $\{0\}$, 
*: the convolution operation on probability distributions.

In addition, the integration of a function $\varphi$ with respect to a measure $\mu$ is denoted by

$$
\int_{\mathbf{R}_{+}} \varphi(x) \mu(d x)=\langle\varphi, \mu\rangle
$$

Let us begin with a renewal argument. Consider a renewal process $\left\{\tau_{j}\right\}_{0 \leq j<\infty}$ with $\tau_{0}=0$ and the inter-occurrence time distribution $\mu_{V}$. Let $N(t):=\sup \left\{j: \tau_{j} \leq t\right\}$ for $t>0$. Let $I$ be a non-negative exponentially distributed random variable with mean $1 / \lambda$. The variable $I$ is assumed to be independent of the renewal process $\left\{\tau_{j}\right\}_{0 \leq j<\infty}$. Let $\mu_{R}$ denote the probability distribution of the random variable $\tau_{N(I)+1}$. Let $V^{*}(s)$ denote the LST of $\mu_{V}$. The LST of the probability distribution $\mu_{R}$ is given by

Lemma 3.1 (e.g. [13]).

$$
E\left[\exp \left(-s \tau_{N(I)+1}\right)\right]=\frac{V^{*}(s)-V^{*}(s+\lambda)}{1-V^{*}(s+\lambda)} .
$$

It is well known that the idle probabilities measured at arrivals, at departures, and at an arbitrary time are the same for the $M / G I / 1 / \infty$ queues whose schedule never refers to the 'future' (e.g. [3]). Then, let $p$ denote the idle probability measured at the customer departure epochs. We use $e$ [resp. 1l] $\in v$ to denote the exhaustive [resp. 1-limited] vacation schedule [7]. Recall that the exhaustive schedule means that the server continues to serve waiting customers until the queue becomes empty, while the 1-limited schedule means that the server starts a new vacation just after each service completion. The goal of this section is to prove the following characterization of $\mathcal{T}$.

Proposition 3.2. The set $\mathcal{T}$ of all interdeparture time distributions $\mu_{T}[\xi], \xi \in \mathcal{V}$, can be characterized as follows:

(i) The set $\mathcal{T}$ is given by a segment with vertices $\left\{\mu_{T}[1 l], \mu_{T}[e]\right\}$, i.e.,

$$
\mathcal{T}=\left\{(1-c) \mu_{T}[1 l]+c \mu_{T}[e] \mid 0 \leq c \leq 1\right\}
$$

(ii) The ratio $c$ for schedule $\xi \in \mathcal{V}$ is given by

$$
c[\xi]=\frac{p[\xi]-p[1 l]}{p[e]-p[1 l]}
$$

where

$$
p[1 l]=\left(\frac{1}{\sigma}-1\right)\left(\frac{1-\rho}{\lambda v}-1\right), \quad p[e]=\frac{(1-\sigma)(1-\rho)}{\lambda v} .
$$

(iii) The vertices $\left\{\mu_{T}[1 l], \mu_{T}[e]\right\}$ are given by

$$
\begin{gathered}
\mu_{T}[1 l]=\mu_{H} *\left((1-p[1 l]) \mu_{V}+p[1 l] \mu_{R}\right), \\
\mu_{T}[e]=\mu_{H} *\left((1-p[e]) \delta_{0}+p[e] \mu_{R}\right) .
\end{gathered}
$$

Remark 3.3. Equalities (3.4) and (3.6) are seen in the literature and not new (see [13]). The emphasis of Proposition 3.2 is laid on the geometric property (3.2) and the relation 
(3.3) between the ratio $c$ and the idle probability $p$. This result means that the parametric structure of the distribution set $\mathcal{T}$ is quite simple. Moreover, the vacation schedule is seen to influence the interdeparture time distribution only through the idle probability.

Proof of Proposition 3.2. Consider an arbitrary customer $A$. The interval between the departures of customer $A$ and the subsequent customer (called $B$ ) consists of the closed interval $J$ beginning with $A^{\prime}$ s departure and ending with the starting epoch of $B^{\prime}$ s service. Let $\mu_{J}$ denote the probability distribution of the length of $J$. Assumption 2.1-(iii) implies that $B^{\prime}$ s service time and the length of $J$ are mutually independent. Thus

$$
\mu_{T}=\mu_{H} * \mu_{J}
$$

Let us determine $\mu_{J}$. There are three disjoint cases at $A^{\prime}$ s departure:

Case 1. The server begins to serve $B$.

Case 2. A new vacation starts and the server leaves behind the customers which have not been served.

Case 3. The system becomes empty.

The probability of Case 3 is given by $p$. Let $q$ denote the probability of Case 2 . The interval $J$ consists of, respectively,

Case 1 , one point ( $A^{\prime}$ s departure time),

Case 2 , the interval of the new vacation,

Case 3 , the idle period and the remaining interval of the vacation which is detected by $B$ at the arrival.

Here, we note that Assumption 2.1-(iv) implies that only one vacation occurs in interval $J$ in Case 2. Now we must determine the length of $J$ in Case 3 . According to the assumption (iv), interval $J$ consists of the vacations started just after $A^{\prime}$ s departure and before $B^{\prime}$ s arrival. Assumption 2.1-(iii) implies that the idle period (the interval beginning with $A^{\prime}$ s departure and ending with $B^{\prime}$ s arrival) is exponentially distributed with mean $1 / \lambda$, and is independent of the i.i.d. sequence of vacations. Hence the length of $J$ in Case 3 is distributed with $\mu_{R}$. Thus

$$
\mu_{J}=(1-p-q) \delta_{0}+q \mu_{V}+p \mu_{R}
$$

i.e.,

$$
\mu_{T}=\mu_{H} *\left((1-p-q) \delta_{0}+q \mu_{V}+p \mu_{R}\right) .
$$

Let us determine $q$. From Lemma 3.1, we have

$$
\int_{\mathbf{R}_{+}} x \mu_{R}(d x)=\frac{v}{1-\sigma} .
$$

Substituting (3.9) and (3.10) into the balance equation

$$
\int_{\mathbf{R}_{+}} x \mu_{T}(d x)=1 / \lambda
$$

we have

$$
q=\frac{1-\rho}{\lambda v}-\frac{1}{1-\sigma} p
$$


Now, (3.4) follows form $(p+q)[1 l]=1, q[e]=0$, and (3.11). Hence (3.5) and (3.6) follow from (3.4), (3.9), and (3.11). From (3.4), (3.5), (3.6), (3.9), and (3.11) for all $\xi \in \mathcal{V}$,

$$
\left(1-\frac{p[\xi]-p[1 l]}{p[e]-p[1 l]}\right) \mu_{J}[1 l]+\left(\frac{p[\xi]-p[1 l]}{p[e]-p[1 l]}\right) \mu_{J}[e]=\mu_{J}[\xi] .
$$

Comparing the coefficients of $\delta_{0}$ in the both sides of this equality, we have,

$$
0 \leq \frac{p[\xi]-p[1 l]}{p[e]-p[1 l]} \leq 1
$$

Hence, we have (3.3) and

$$
\mathcal{T} \subset\left\{(1-c) \mu_{T}[1 l]+c \mu_{T}[e] \mid 0 \leq c \leq 1\right\} .
$$

To complete the proof, we must show that for a given, $c, 0 \leq c \leq 1$, there exits a schedule $\xi_{c} \in \mathcal{V}$ satisfying

$$
c=\frac{p\left[\xi_{c}\right]-p[1 l]}{p[e]-p[1 l]}
$$

This is accomplished by the Bernoulli schedule [10]. Let us describe the schedule. Consider again $A^{\prime}$ s departure. If the system becomes empty (Case 3 ), the subsequent service process is uniquely determined by Assumption 2.1-(iv). Now, consider the complementary case. The occurrences of Case 1 and Case 2 are determined by the vacation schedule. A Bernoulli schedule chooses these two cases according to a Bernoulli sequence. Let $b, 0 \leq b \leq 1$, be the probability that Case 1 is chosen. The idle probability under this schedule is given by

$$
p=1-\frac{1-p[e]}{\sigma+(1-\sigma) b}
$$

which is monotonically increasing in $b$. The value of the right-hand side at $b=0$ [resp. $b=1]$ is equal to $p[1 l][$ resp. $p[e]]$. Hence, for a given $c, 0 \leq c \leq 1$, there exists $b, 0 \leq b \leq 1$, such that (3.12) holds with $\xi_{c}$ assigned to the Bernoulli schedule with parameter $b$. Thus we have

$$
\mathcal{T} \supset\left\{(1-c) \mu_{T}[1 l]+c \mu_{T}[e] \mid 0 \leq c \leq 1\right\}
$$

Remark 3.4. For a stationary and stable $G / G I / 1 / \infty$ queue with i.i.d. vacations, the above argument is available except for the idle time distribution. That is, let $I_{G}$ be the idle time measured at a customer departure. The equalities

$$
\begin{gathered}
\left.\mu_{T}=\mu_{H} *\left((1-p-q) \delta_{0}+q \mu_{V}+p\left(\delta_{0}-\mu_{V}\right) * F_{G} \eta_{V}\right)\right) \\
q=\frac{1-\rho}{\lambda v}-\frac{r}{v} p
\end{gathered}
$$

can be obtained similarly, where

$$
\begin{aligned}
& F_{G}(x):=P\left[I_{G} \leq x\right] \\
& \left(F_{G} \eta_{V}\right)(d x):=F_{G}(x) \eta_{V}(d x) \\
& r:=\int_{\mathbf{R}_{+}} x\left(\left(\delta_{0}-\mu_{V}\right) *\left(F_{G} \eta_{V}\right)\right)(d x),
\end{aligned}
$$

$\eta_{V}$ : the measure on $\mathbf{R}_{+}$associated with the renewal process $\left\{\tau_{j}\right\}_{0 \leq j<\infty}$ 
defined by

$$
\eta_{V}((s, t])=E[N(t)-N(s)] \text { for each } 0<s \leq t<+\infty, \eta_{V}(\{0\})=0 .
$$

The idle time distribution seems difficult to characterize in general and seems sensitive to the vacation schedule $\xi$ if the arrival process is not Poissonian. The parametric property in Proposition 3.2 is a consequence of the insensitivity that the idle time is independent of the schedule $\xi$ if the arrival process is Poissonian.

\section{Ordering properties of the distribution set $\mathcal{T}$}

This section investigates the ordered structure on the set $\mathcal{T}$ to describe how the variability of interdeparture times depends on the vacation schedule and the length of vacations.

Let us recall the notion of the ordering of probability distributions (see e.g. [12]). Let $\mathcal{F}_{c x}$ [resp. $\mathcal{F}_{i c x}$ ] be the set of all convex (measurable) functions [resp. increasing and convex functions] on non-negative real numbers $\mathbf{R}_{+}$. Let $\mu$ and $\nu$ be probability distributions on $\mathbf{R}$. The distribution $\mu$ is said to be larger than $\nu$ in the convex order [resp. increasing convex order], denoted by $\mu \leq_{c x} \nu$ [resp. $\left.\mu \leq_{i c x} \nu\right]$, if and only if $\langle\varphi, \mu\rangle \leq\langle\varphi, \nu\rangle$ for all $\varphi \in \mathcal{F}_{c x}\left[\right.$ resp. $\left.\varphi \in \mathcal{F}_{i c x}\right]$, where, for divergent cases, the inequality means that if $\langle\varphi, \nu\rangle=$ $+\infty[$ resp. $\langle\varphi, \mu\rangle=-\infty]$ then $\langle\varphi, \mu\rangle=+\infty[$ resp. $\langle\varphi, \nu\rangle=-\infty]$.

The following theorem describes how the variability depends on the vacation schedule.

Theorem 4.1. For a given triplet $\left(\lambda, \mu_{H}, \mu_{V}\right)$ satisfying the simultaneous stability condition (2.1),

$\left(1-c_{1}\right) \mu_{T}[1 l]+c_{1} \mu_{T}[e] \leq_{c x}\left(1-c_{2}\right) \mu_{T}[1 l]+c_{2} \mu_{T}[e]$ if and only if $c_{1} \leq c_{2}$ for all $c_{1}, c_{2} \in[0,1]$.

Remark 4.2. Together with the parametric property in Proposition 3.2, Theorem 4.1 means that the ordered space $\left(\mathcal{T}, \leq_{c x}\right)$ can be naturally identified with the standard interval $([0,1], \leq)$. Hence, the order $\leq_{c x}$ is total (i.e., all pairs $(\mu, \nu) \mu, \nu \in \mathcal{T}$ are comparable) and

$$
\mu_{T}[1 l]=\inf _{\leq x} \mathcal{T}, \quad \mu_{T}[e]=\sup _{\leq c x} \mathcal{T}
$$

Moreover, let $e(k)[$ resp. $g(k)] \in \mathcal{V}$ be the $k$-limited exhaustive [resp. gated] vacation schedule [7]. Note that $e=e(\infty), 1 l=e(1)=g(1)$. It is shown that for $k=1,2,3, \cdots$,

$$
p[e(k)] \leq p[e(k+1)], p[g(k)] \leq p[g(k+1)], \text { and }[p(k)] \leq p[e(k)]
$$

in a manner similar to Tedijanto [14] (by which the first assertion was treated). From (3.3), for $k=1,2,3, \cdots$,

$$
\mu_{T}[e(k)] \leq_{c x} \mu_{T}[e(k+1)], \mu_{T}[g(k)] \leq_{c x} \mu_{T}[g(k+1)], \mu_{T}[g(k)] \leq_{c x} \mu_{T}[e(k)],
$$

and consequently, for $k=1,2,3, \cdots$,

$$
C_{T}[e(k)] \leq C_{T}[e(k+1)], C_{T}[g(k)] \leq C_{T}[g(k+1)], C_{T}[g(k)] \leq C_{T}[e(k)],
$$

where $C_{T}$ denotes the coefficient of vacation of the interdeparture time.

For convenience, the following notation will be used

$$
\exp _{\lambda}(x):=e^{-\lambda x}
$$




$$
M_{\lambda}(d x):=\lambda e^{-\lambda x} d x .
$$

Proof of Theorem 4.1. Put $\Gamma(c):=(1-c) \mu_{T}[1 l]+c \mu_{T}[e]$ for $0 \leq c \leq 1$. Let $c_{1}, c_{2} \in[0,1]$. Because the ordered space $([0,1], \leq)$ is total and the mapping $\Gamma:[0,1] \rightarrow \mathcal{T}$ is bijective from (3.2), it suffices to show that $\Gamma\left(c_{1}\right) \leq_{c x} \Gamma\left(c_{2}\right)$. Put $\Pi(c):=(1-c) \mu_{J}[1 l]+C \mu_{J}[e]$ (see (3.8)) for $0 \leq c \leq 1$. From $\Gamma(c)=\mu_{H} * \Pi(c)$, we have

$$
\langle\varphi, \Gamma(c)\rangle=\left\langle\varphi(\bullet+\bullet), \mu_{H} \otimes \Pi(c)\right\rangle,
$$

where $\varphi(\bullet+\bullet)$ denotes the function $(x, y) \mapsto \varphi(x+y)$. Note that the function $y \mapsto\langle\varphi(y+$ -), $\left.\mu_{H}\right\rangle$ belongs to $\mathcal{F}_{c x}$. Now, it suffices to show $\Pi\left(c_{1}\right) \leq_{c x} \Pi\left(c_{2}\right)$ which is equivalent to

$$
\left\langle\varphi, \Pi\left(c_{1}\right)\right\rangle \leq\left\langle\varphi, \Pi\left(c_{2}\right)\right\rangle, \text { for all } \varphi \in \mathcal{F}_{c x} .
$$

Here, we may assume $\varphi(0)=0$. From (3.4),(3.5), and (3.6), we have

$$
\left\langle\varphi, \Pi\left(c_{2}\right)-\Pi\left(c_{1}\right)\right\rangle=\left(c_{2}-c_{1}\right)(p[e]-p[1 l])\left(\left\langle\varphi, \mu_{R}\right\rangle-\frac{1}{1-\sigma}\left\langle\varphi, \mu_{V}\right\rangle\right),
$$

for all $\varphi \in \mathcal{F}_{c x}$ satisfying $\varphi(0)=0$. Hence (4.3) is reduced to the inequality

$$
\left\langle\varphi, \mu_{R}\right\rangle \geq \frac{1}{1-\sigma}\left\langle\varphi, \mu_{V}\right\rangle, \text { for all } \varphi \in \mathcal{F}_{c x} \text { satisfying } \varphi(0)=0 .
$$

For convenience, we consider $\tilde{\eta}_{V}:=\delta_{0}+\eta_{V}$. From the renewal theory (e.g. [2]), $\tilde{\eta}_{V}$ is characterized by

$$
\tilde{\eta}_{V}=\delta_{0}+\mu_{V} * \tilde{\eta}_{V}
$$

and hence

$$
\left\langle\exp _{s}, \tilde{\eta}_{V}\right\rangle=\frac{1}{1-V^{*}(s)} .
$$

From (3.1), (4.6) implies

$$
\mu_{R}=\left(\exp _{\lambda} \tilde{\eta}_{V}\right) *\left(\left(1-\exp _{\lambda}\right) \mu_{V}\right) .
$$

On the other hand, as the derivative of $\varphi$ is monotonically increasing,

$$
\varphi(x+y) \geq \varphi(x)+\varphi(y) .
$$

Hence,

$$
\begin{aligned}
\left\langle\varphi, \mu_{R}\right\rangle & =\left\langle\varphi,\left(\exp _{\lambda} \tilde{\eta}_{V}\right) *\left(\left(1-\exp _{\lambda}\right) \mu_{V}\right)\right\rangle \quad(\text { by }(4.7)) \\
& \geq \int_{\mathbf{R}_{+} \times \mathbf{R}_{+}}(\varphi(x)+\varphi(y)) e^{-\lambda x} \tilde{\eta}_{V}(d x)\left(1-e^{-\lambda y}\right) \mu_{V}(d y) \quad(\text { by }(4.8)) \\
& =\left\langle\varphi \exp _{\lambda}, \tilde{\eta}_{V}\right\rangle\left\langle 1-\exp _{\lambda}, \mu_{V}\right\rangle+\left\langle\varphi\left(1-\exp _{\lambda}\right), \mu_{V}\right\rangle\left\langle\exp _{\lambda}, \tilde{\eta}_{V}\right\rangle
\end{aligned}
$$

From (4.6), we have

$$
\left\langle\varphi, \mu_{R}\right\rangle \geq(1-\sigma)\left\langle\varphi \exp _{\lambda}, \tilde{\eta}_{V}\right\rangle+\frac{1}{1-\sigma}\left\langle\varphi\left(1-\exp _{\lambda}\right), \mu_{V}\right\rangle
$$


Substituting (4.5) and $\varphi(0)=0$ into $\left\langle\varphi \exp _{\lambda}, \tilde{\eta}_{V}\right\rangle,(4.8)$ also implies

$$
\begin{aligned}
& \left\langle\varphi \exp _{\lambda}, \tilde{\eta}_{V}\right\rangle=\left\langle\varphi \exp _{\lambda}, \tilde{\eta}_{V} *\left(\exp _{\lambda} \mu_{V}\right)\right\rangle \\
& \geq\left\langle\exp _{\lambda}, \mu_{V}\right\rangle\left(\varphi \exp _{\lambda}, \tilde{\eta}_{V}\right\rangle+\left\langle\exp _{\lambda}, \tilde{\eta}_{V}\right\rangle\left\langle\varphi \exp _{\lambda} \mu_{V}\right\rangle \\
& =\sigma\left\langle\varphi \exp _{\lambda}, \tilde{\eta}_{V}\right\rangle+\frac{1}{1-\sigma}\left\langle\varphi \exp _{\lambda}, \mu_{V}\right\rangle .
\end{aligned}
$$

Hence,

$$
\left\langle\exp _{\lambda}, \tilde{\eta}_{V}\right\rangle \geq \frac{1}{(1-\sigma)^{2}}\left\langle\varphi \exp _{\lambda}, \mu_{V}\right\rangle
$$

Substituing (4.10) into (4.9), we have

$$
\left\langle\varphi, \mu_{R}\right\rangle \geq \frac{(1-\sigma)}{(1-\sigma)^{2}}\left\langle\varphi \exp _{\lambda}, \mu_{V}\right\rangle+\frac{1}{1-\sigma}\left\langle\varphi\left(1-\exp _{\lambda}\right), \mu_{V}\right\rangle=\frac{1}{1-\sigma}\left\langle\varphi, \mu_{V}\right\rangle
$$

This completes the proof of (4.4).

In view of the application described in Section 1, the vacation time may be short. In this case, we can describe how the variability depends on the length of vacation with the aid of the infinitesimal calculus. For a given triplet $\left(\lambda, \mu_{H}, \mu_{V}\right)$, we consider a perturbation $\left(\lambda, \mu_{H}, \mu_{V}^{\varepsilon}\right)$ with a small $\varepsilon \geq 0$ from the ordinary $M / G / 1$ queue characterized by $\left(\lambda, \mu_{H}\right)$. That is, we define $\mu_{V}^{\varepsilon}$ by

$$
\left\langle\varphi, \mu_{V}^{\varepsilon}\right\rangle=\int_{\mathbf{R}_{+}} \varphi(\varepsilon x) \mu_{V}(d x) .
$$

For a quantity $Q$ defined for $\left(\lambda, \mu_{H}, \mu_{V}\right), Q^{\varepsilon}$ denotes the corresponding quantity defined for $\left(\lambda, \mu_{H}, \mu_{V}^{\varepsilon}\right)$. For $\varepsilon=0, \mu_{T}^{0}=\rho \mu_{H}+(1-\rho) \mu_{H} * M_{\lambda}$, which is the interdeparture time distribution of the ordinary $M / G / 1$ queue. Let $\alpha$ be the probability that the server leaves behind no customers at an arbitrary vacation epoch. For a schedule $\xi \in \mathcal{V}$, consider the expansion

$$
\alpha^{\varepsilon}=1-\beta \cdot \lambda v \varepsilon+o(\varepsilon) \text {, as } \varepsilon \rightarrow+0 .
$$

For the schedule with $\beta[\xi]=0$, we further consider

$$
\alpha^{\varepsilon}=1-\gamma(\lambda v \varepsilon)^{2}+o\left(\varepsilon^{2}\right), \text { as } \varepsilon \rightarrow+0 .
$$

Since $\alpha^{\varepsilon} \leq 1$, we have $\beta, \gamma \geq 0$. Let $\mu_{\tilde{V}}$ denote the recurrence time distribution of the vacation time defined by $\mu_{\tilde{V}}(d x):=\frac{1}{v} \mu_{V}[x, \infty) d x$, and let $C^{k}$ denote the set of all functions $\varphi$ on strictly positive real numbers having a continuous $k$-th derivative $\varphi^{(k)}$ and a limit $\varphi^{(k)}(0+)$. We now present how the variability depends on the length of vacations.

Proposition 4.3. For a quadruplet $\left(\lambda, \mu_{H}, \mu_{V}, \xi\right)$, the perturbations $\left\{\mu_{T}^{\varepsilon}\right\}_{\varepsilon \geq 0}$ are comparable for small $\varepsilon$ in the following sence: for $\psi \in \mathcal{F}_{i c x} \cap C^{3}$ with

$$
\left\langle\psi+\psi^{\prime}, \mu_{\tilde{V}} * M_{\lambda} * \mu_{H}\right\rangle+\left\langle\psi, \mu_{V} * \mu_{H}\right\rangle<\infty
$$

(i) if $\beta[\xi]>0$, then there exits $a>0$ such that

$$
\left\langle\psi, \mu_{T}^{\varepsilon_{1}}[\xi]\right\rangle \leq\left\langle\psi, \mu_{T}^{\varepsilon_{2}}[\xi]\right\rangle \text { for all } 0 \leq \varepsilon_{2} \leq \varepsilon_{1} \leq a
$$


(ii) if $\beta[\xi]=0$ and $\frac{v^{(3)}}{3 v^{3}}-\left(\frac{v^{(2)}}{2 v^{2}}\right)^{2}<$ [resp. $\left.>\right] \gamma[\xi]$, then there exists $a>0$ such that

$$
\left\langle\psi, \mu_{T}^{\varepsilon_{1}}[\xi] \leq[\text { resp. } \geq]\left\langle\psi, \mu_{T}^{\varepsilon_{2}}[\xi]\right\rangle \text { for all } 0 \leq \varepsilon_{2} \leq \varepsilon_{1} \leq a\right.
$$

We note that the assumption for $\psi$ is used to apply Lemma 4.5 , where the condition is used to guarantee convergences of the terms produced by the integration by parts.

Remark 4.4. For the typical vacation schedules, the coefficients in the expansions (4.11) and (4.12) are given by

$$
\beta[e]=0, \gamma[e]=0, \beta[g]=\sum_{n=0}^{\infty}\left(1-u_{n}\right) \text { and } \beta[1 l]=\frac{\rho}{1-\rho}
$$

where $g \in \mathcal{V}$ denotes the gate schedule characterized by $g=g(\infty)$, the sequence $\left\{u_{n}\right\}_{n=0}^{\infty}$ is defined inductively by $u_{0}:=H^{*}(\lambda), u_{n}:=H^{*}\left(\lambda-\lambda u_{n-1}\right)$ for $n=1,2,3 \cdots$ (see [6]). More generally, from $(4.2)$ and $p=(1-\rho)(1-\sigma) \alpha / \lambda v([6])$,

$$
\beta[e(k+1)] \leq \beta[e(k)], \beta[g(k+1)] \leq \beta[g(k)] \text { for } k=1,2,3 \cdots
$$

Because of Schwartz's inequality, $\frac{v^{(3)}}{3 v^{3}}-\left(\frac{v^{(2)}}{2 v^{2}}\right)^{2}>0$ (if $v^{(2)}<\infty$ ). Thus Proposition 4.3 implies for all $\psi \in \mathcal{F}_{i c x} \cap C^{3}$ with $\left\langle\psi, \mu_{\tilde{v}} * M_{\lambda} * \mu_{H}\right\rangle<\infty$

$$
\left\langle\psi, \mu_{T}[1 l]\right\rangle \leq\left\langle\psi, \mu_{T}[g]\right\rangle \leq\left\langle\psi, \mu_{T}^{0}\right\rangle \leq\left\langle\psi, \mu_{T}[e]\right\rangle
$$

if the vacation times are very short. The range of vacation times under which (4.17) above hold may depend on the function $\psi$.

To prove Proposition 4.3, we need the asymptotic expansion of the integration $\left\langle\varphi, \mu_{R}^{\varepsilon}\right\rangle$.

Lemma 4.5. If $\varphi \in \mathcal{F}_{i c x} \cap C^{3}$ with $\varphi(0)=\varphi^{\prime}(+0)=0$, and $\left\langle\varphi+\varphi^{\prime}, \mu_{\tilde{V}} * M_{\lambda}\right\rangle<\infty$, then $\left\langle|\varphi|, \mu_{R}^{\varepsilon}\right\rangle<\infty$ for all $0 \leq \varepsilon \leq 1$ and

$$
\left\langle\varphi, \mu_{R}^{\varepsilon}\right\rangle=\left\langle\varphi, M_{\lambda}\right\rangle\left(1+\frac{v^{(2)}}{2 v^{2}} \lambda v \varepsilon+\frac{v^{(3)}}{6 v^{3}}(\lambda v \varepsilon)^{2}\right)+o\left(\varepsilon^{2}\right), \text { as } \varepsilon \rightarrow+0 .
$$

Proof of Proposition 4.3. Consider again the equality $\mu_{T}=\mu_{H} * \mu_{J}$ as described in the proof of Proposition 3.2. Because $\left\langle\psi, \mu_{T}\right\rangle=\left\langle\psi(\bullet+\bullet), \mu_{H} \otimes \mu_{J}\right\rangle$, it suffices to show (4.13) and (4.14) for $\left\langle\psi_{0}, \mu_{J}^{\varepsilon}\right\rangle$ instead of $\left\langle\psi, \mu_{T}^{\varepsilon}\right\rangle$ where

$$
\begin{gathered}
\psi_{0}(y)=\left\langle\psi(\bullet+y), \mu_{H}\right\rangle \in \mathcal{F}_{i c x} \cap C^{3}, \\
\mu_{J}^{0}=\rho \delta_{0}+(1-\rho) M_{\lambda}
\end{gathered}
$$

Note that

$$
\left\langle\psi_{0}+\psi_{0}^{\prime}, \mu_{\tilde{V}} * M_{\lambda}\right\rangle+\left\langle\psi_{0}, \mu_{V}\right\rangle<\infty .
$$

Put $\varphi(x):=\psi_{0}(x)-\psi_{0}(0)-\psi_{0}^{\prime}(+0) x$. Then $\varphi \in \mathcal{F}_{i c x} \cap C^{3}, \varphi(0)=\varphi^{\prime}(+0)=0$, and

$$
\left\langle\varphi+\varphi^{\prime}, \mu_{\tilde{V}} * M_{\lambda}\right\rangle+\left\langle\varphi, \mu_{V}\right\rangle<\infty
$$


The equality

$$
\left\langle\varphi, \mu_{J}^{\varepsilon}\right\rangle=\left\langle\psi_{0}, \mu_{J}^{\varepsilon}\right\rangle-\psi_{0}(0)-\psi_{0}^{\prime}(+0)(1 / \lambda-h),
$$

implies that $\left|\left\langle\psi, \mu_{T}^{\varepsilon}\right\rangle\right|<\infty$ (Lemma 4.5) and that it suffices to compare $\left\langle\varphi, \mu_{J}^{\varepsilon}\right\rangle$. From the Fuhrmann-Cooper decomposition $[6], p=(1-\rho)(1-\sigma) \alpha / \lambda v$. Then we have

$$
\begin{aligned}
& p^{\varepsilon}=(1-\rho)\left(1-\frac{v^{(2)}}{2 v^{2}} \lambda v \varepsilon+\frac{v^{(3)}}{6 v^{3}}(\lambda v \varepsilon)^{2}\right)\left(1-\beta \lambda v \varepsilon-\gamma(\lambda v \varepsilon)^{2}\right)+o\left(\varepsilon^{2}\right) \\
& q^{\varepsilon}=(1-\rho)(\beta+\gamma \lambda v \varepsilon)+o(\varepsilon)
\end{aligned}
$$

Therefore,

$$
\begin{aligned}
\left\langle\varphi, \mu_{J}^{\varepsilon}\right\rangle= & q^{\varepsilon}\left\langle\varphi, \mu_{V}^{\varepsilon}\right\rangle+p^{\varepsilon}\left\langle\varphi, \mu_{R}^{\varepsilon}\right\rangle \\
= & (1-\rho)(\beta+\gamma \lambda v \varepsilon+o(\varepsilon)) o(\varepsilon)+p^{\varepsilon}\left\langle\varphi, \mu_{R}^{\varepsilon}\right\rangle \\
= & (1-\rho)\left(1-\frac{v^{(2)}}{2 v^{2}} \lambda v \varepsilon+\frac{v^{(3)}}{6 v^{3}}(\lambda v \varepsilon)^{2}\right)\left(1-\beta \lambda v \varepsilon-\gamma(\lambda v \varepsilon)^{2}\right)\left\langle\varphi, \mu_{R}^{\varepsilon}\right\rangle \\
& \quad+\beta o(\varepsilon)+o\left(\varepsilon^{2}\right) .
\end{aligned}
$$

From Lemma 4.5, we have

$$
\left\langle\varphi, \mu_{J}^{\varepsilon}\right\rangle=\left\langle\varphi, M_{\lambda}\right\rangle(1-\rho)(1-\beta \cdot \lambda v \varepsilon)+o(\varepsilon)
$$

as $\varepsilon \rightarrow+0$. If $\beta[\xi]=0$,

$$
\left\langle\varphi, \mu_{J}^{\varepsilon}\right\rangle=\left\langle\varphi, M_{\lambda}\right\rangle(1-\rho)\left(1+\left(\frac{v^{(3)}}{3 v^{3}}-\left(\frac{v^{(2)}}{2 v^{2}}\right)^{2}-\gamma\right) \cdot(\lambda v \varepsilon)^{2}\right)+o\left(\varepsilon^{2}\right)
$$

as $\varepsilon \rightarrow+0$. Assume $\beta[\xi]>0$ or $\frac{v^{(3)}}{3 v^{3}}-\left(\frac{v^{(2)}}{2 v^{2}}\right)<[$ resp. $>] \gamma[\xi]$. There exists $a>0$ such that $\left\langle\varphi, \mu_{J}^{\varepsilon}\right\rangle$ is non-increasing [resp. non-decreasing] in $\varepsilon \in[0, a]$. This completes the proof.

Proof of Lemma 4.5. Consider the renewal process $\left\{\tau_{j}\right\}_{0 \leq j<\infty}$ used for the definition of $\mu_{R}$. For $x \geq 0$, let $\theta_{x}$ denote the time shift defined by $\tau_{j} \circ \theta_{x}=\tau_{N(x)+j}-x$ for $j=0,1,2, \cdots$. By definition

$$
\left\langle\varphi, \mu_{R}\right\rangle=E\left[\varphi\left(\tau_{N(I)+1}\right)\right]=E\left[\int_{\mathbf{R}_{+}} \varphi\left(\tau_{N(x)+1}\right) M_{\lambda}(d x)\right]=E\left[\int_{\mathbf{R}_{+}} \varphi\left(\tau_{1} \circ \theta_{x}+x\right) M_{\lambda}(d x)\right] .
$$

Note that $\varphi, \varphi^{\prime} \geq 0$ because $\varphi \in \mathcal{F}_{i c x}$ and $\varphi(0)=\varphi^{\prime}(0)=0$. Let us first show $\left\langle\varphi, \mu_{R}^{\varepsilon}\right\rangle<\infty$. From integration by parts,

$$
\begin{aligned}
& E\left[\int_{\mathbf{R}_{+}} \varphi\left(\tau_{1} \circ \theta_{x}+x\right) M_{\lambda}(d x)\right]=\lim _{x \rightarrow \infty}\left(\lambda x e^{-\lambda x}\right)\left(1 / x \int_{0}^{x} E\left[\varphi\left(\tau_{1} \circ \theta_{y}+y\right)\right] d y\right) \\
&+\lambda \lim _{u \rightarrow \infty} \int_{0}^{u}\left(\lambda x e^{-\lambda x}\right)\left(1 / x \int_{0}^{x} E\left[\varphi\left(\tau_{1} \circ \theta_{y}+y\right)\right] d y\right) d x
\end{aligned}
$$

By the ergodicity of the renewal process and Theorem 1.3.12 in [5], the first term is given by

$$
\lim _{x \rightarrow \infty}\left(\lambda x e^{-\lambda x}\right)\left(1 / x \int_{0}^{x} E\left[\varphi\left(\tau_{1} \circ \theta_{y}+y\right)\right] d y\right)=\lim _{x \rightarrow \infty}\left(\lambda e^{-\lambda x}\right) \int_{0}^{x}\left\langle\varphi(u+y), \mu_{\tilde{V}}(d u)\right\rangle d y
$$


The right-hand side is finite (it converges to 0 ) as $\left\langle\varphi^{\prime}, \mu_{\tilde{V}} * M_{\lambda}\right\rangle<\infty$. Similarly, the convergence of the second term is identical to the convergence of the integration

$$
\begin{aligned}
& \lambda \lim _{z \rightarrow \infty} \int_{0}^{z}\left(\lambda e^{-\lambda x}\right)\left(\int_{0}^{x}\left\langle\varphi(u+y), \mu_{\tilde{V}}(d u)\right\rangle d y\right) d x \\
& =-\lim _{x \rightarrow \infty}\left(\lambda e^{-\lambda x}\right) \int_{0}^{x}\left\langle\varphi(u+y), \mu_{\tilde{V}}(d u)\right\rangle d y+\left\langle\varphi, \mu_{\tilde{V}} * M_{\lambda}\right\rangle .
\end{aligned}
$$

Hence $\left\langle\varphi, \mu_{R}\right\rangle<\infty$. As $\varphi$ is non-decreasing, this implies $\left\langle\varphi, \mu_{R}^{\varepsilon}\right\rangle<\infty$ for all $0<\varepsilon \leq 1$ while the case of $\varepsilon=0$ is trivial. Now, we show the asymptotic properties. Applying Taylor's expansion to (4.21),

$$
\begin{aligned}
& (4.22)\left\langle\varphi, \mu_{R}^{\varepsilon}\right\rangle=\left\langle\varphi, M_{\lambda}\right\rangle+\varepsilon E\left[\left\langle\left(\tau_{1} \circ \theta_{(\bullet / \varepsilon)}\right) \varphi^{\prime}, M_{\lambda}\right\rangle\right] \\
& \quad+\varepsilon^{2} E\left[\left\langle\left(\tau_{1} \circ \theta_{(\bullet / \varepsilon)}\right)^{2} \frac{1}{2} \varphi^{\prime \prime}, M_{\lambda}\right\rangle\right]+\varepsilon^{3} E\left[\left\langle\left(\int_{0}^{\tau_{1}} \varphi^{(3)}(\varepsilon y+\bullet) \frac{1}{2}\left(\tau_{1}-y\right)^{2} d y\right) \circ \theta_{(\bullet / \varepsilon)}, M_{\lambda}\right\rangle\right] .
\end{aligned}
$$

Let us estimate the second term. Put $F(x):=1-e^{-\lambda x}=1-\exp _{\lambda}(x)$. Integration by parts yields

$$
\left\langle\left(\tau_{1} \circ \theta_{(\bullet / \varepsilon)}\right) \varphi^{\prime}, M_{\lambda}\right\rangle=-\int_{\mathbf{R}_{+}} \varepsilon \int_{0}^{x / \varepsilon}\left(\tau_{1} \circ \theta_{y}\right) d y\left(\varphi^{\prime}(x) F^{\prime}(x)\right)^{\prime} d x .
$$

Using a similar ergodic argument,

$$
\lim _{\varepsilon \rightarrow 0+} \varepsilon \int_{0}^{x / \varepsilon}\left(\tau_{1} \circ \theta_{y}\right) d y=\frac{v^{(2)}}{2 v} x .
$$

Put $g(x)=\int_{0}^{x}\left(\tau_{1} \circ \theta_{y}\right) d y-\frac{v^{(2)}}{2 v} x$. Integration by parts again yields

$$
\begin{aligned}
& \left\langle\left(\tau_{1} \circ \theta_{(\bullet / \varepsilon)}\right) \varphi^{\prime}, M_{\lambda}\right\rangle=-\int_{\mathbf{R}_{+}}\left(\varepsilon g(x / \varepsilon)+\frac{v^{(2)}}{2 v} x\right)\left(\varphi^{\prime}(x) F^{\prime}(x)\right)^{\prime} d x \\
& =\frac{v^{(2)}}{2 v}\left\langle\varphi^{\prime}, M_{\lambda}\right\rangle-\varepsilon \int_{\mathbf{R}_{+}} \varepsilon \int_{0}^{x / \varepsilon} g(y) d y\left(\varphi^{\prime}(x) F^{\prime}(x)\right)^{\prime \prime} d x, \\
& \lim _{\varepsilon \rightarrow 0+} \int_{\mathbf{R}_{+}} \varepsilon \int_{0}^{x / \varepsilon} g(y) d y\left(\varphi^{\prime}(x) F^{\prime}(x)\right)^{\prime \prime} d x=\left(\lim _{t \rightarrow \infty} \frac{1}{t} \int_{0}^{t} g(y) d y\right) \int_{\mathbf{R}_{+}} x\left(\varphi^{\prime}(x) F^{\prime}(x)\right)^{\prime \prime} d x \\
& =-\left(\lim _{t \rightarrow \infty} \frac{1}{t} \int_{0}^{t} g(y) d y\right) \int_{\mathbf{R}_{+}}\left(\varphi^{\prime}(x) F^{\prime}(x)\right)^{\prime} d x=\left(\lim _{t \rightarrow \infty} \frac{1}{t} \int_{0}^{t} g(y) d y\right)\left(\lambda \varphi^{\prime}(0)\right)=0, \\
& \left\langle\varphi^{\prime}, M_{\lambda}\right\rangle=\lambda\left\langle\varphi, M_{\lambda}\right\rangle .
\end{aligned}
$$

These equalities yield,

$$
\varepsilon E\left[\left\langle\left(\tau_{1} \circ \theta_{(\bullet / \varepsilon)}\right) \varphi^{\prime}, M_{\lambda}\right\rangle\right]=\frac{v^{(2)}}{2 v^{2}}\left\langle\varphi, M_{\lambda}\right\rangle \lambda v \varepsilon+o\left(\varepsilon^{2}\right) .
$$

Similarly,

$$
\begin{aligned}
& \varepsilon^{2} E\left[\left\langle\left(\tau_{1} \circ \theta_{(\bullet / \varepsilon)}\right)^{2} \frac{1}{2} \varphi^{\prime \prime}, M_{\lambda}\right\rangle\right]=\frac{1}{6} \frac{v^{(3)}}{v^{3}}\left\langle\varphi, M_{\lambda}\right\rangle(\lambda v \varepsilon)^{2}+o\left(\varepsilon^{2}\right), \\
& \lim _{\varepsilon \rightarrow 0+}\left|E\left[\left\langle\left(\int_{0}^{\tau_{1}} \varepsilon^{(3)}(\varepsilon y+\bullet) \frac{1}{2}\left(\tau_{1}-y\right)^{2} d y\right) \circ \theta_{(\bullet / \varepsilon)}, M_{\lambda}\right\rangle\right]\right| \\
& \leq\left\langle\varphi, \mu_{\tilde{V}} * M_{\lambda}\right\rangle+\left(1+\frac{v^{(2)}}{2 v^{2}} \lambda v+\frac{v^{(3)}}{6 v^{3}}(\lambda v)^{2}\right)\left\langle\varphi, M_{\lambda}\right\rangle<\infty .
\end{aligned}
$$


Substitution of (4.23) through (4.25) into (4.22) yields (4.18).

Finally, let us summarize the ordering property on the perturbed sets $\bigcup_{\varepsilon \text { near } 0} \mathcal{T}^{\varepsilon}$ where the set $\mathcal{T}^{\varepsilon}$ converges to one point $\left\{\mu_{T}^{0}\right\}$ as $\varepsilon \rightarrow 0$. Theorem 4.1 and Proposition 4.3 can be combined and represented as Fig. 1. For an increasing convex function $\psi$, consider the function $\langle\varphi, \bullet\rangle: \bigcup_{\varepsilon \text { near } 0} \mathcal{T}^{\varepsilon} \ni \mu \mapsto\langle\psi, \mu\rangle$. The function $\langle\psi, \bullet\rangle$ increases along the directions represented by arrows in Fig. 1, whereas the function is constant along the dotted curve.

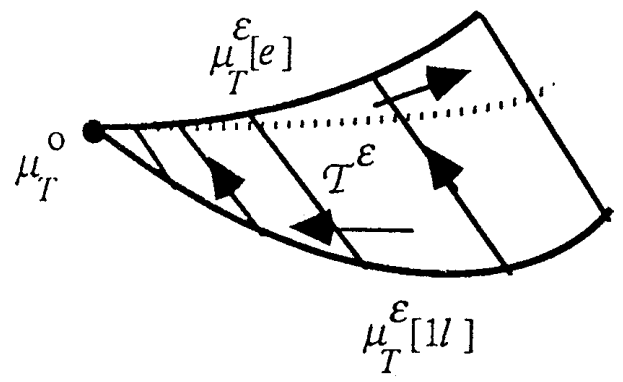

Fig. 1. The increasing convex order of interdeparture time distributions.

\section{Concluding discussion}

Here, we interpret the results in terms of the LANs. Consider the data transfer between terminals in the lightly-loaded token-passing LAN as described in Section 1. The maximum time during which a terminal can transmit packets consecutively is called the token holding time [1] of a terminal. The number $k$ in the vacation model with the $k$-limited schedule may correspond to the token holding time of the source terminal whereas the increase in the vacation time means that the traffic intensities or the token holding times increase at other terminals. Based on the studies of the set of interdeparture time distributions, we have the following consequences.

- The inter-arrival times of packets to the destination becomes less variable if the token holding time at the source terminal is reduced (Theorem 4.1).

- This effect is accelerated if the traffic intensities or the token holding times increase at other terminals ((4.16) and (4.19)).

- An increase in the traffic intensities or the token holding times of other terminal makes the inter-arrival times less variable for most transmission schedules, whereas this effect is accelerated if the token holding time at the source terminal is sufficiently restricted (Proposition 4.3 and $(4.19))$.

Here, the traffic intensities and the token holding times of other terminals are limited to be sufficiently small values because Proposition 4.3 and (4.19) are asymptotic results. These results indicate that the variability of the arrival process and hence the communication quality may behave similarly. Further studies in departure processes are expected to confirm the quality behaviors.

Finally we note the disadvantages of choosing the interdeparture time distribution as the characteristic of the departure processes. First of all, we cannot apply those results directly to the performance under medium, heavy, or bursty traffic environments. This is because the 
inter-arrival times may be correlated so strong under these traffic environments that the interarrival time distribution is inadequate to characterize the number of arrivals in time intervals. On the other hand, according to the proof of Proposition 3.2, the set of inter-departure time distribution $\mathcal{T}$ can be completely parametrized by the Bernoulli schedules. Therefore, the important differences between the $k$-limited schedule and the Bernoulli schedules are not described in this study.

\section{Acknowledgments}

We are grateful to Professor On Hashida of the University of Tsukuba and Dr. Fumiaki Machihara of the NTT Telecommunication Networks Laboratories for their continuous encouragement during this work.

\section{References}

[1] Bux, W., Local area subnetworks: A performance comparison, IEEE Trans. Commun., Vol. COM-29, pp. 1465-1473, 1981.

[2] Cinlar, E., Introduction to Stochastic Processes. Prentice Hall, 1975.

[3] Cooper, R. B., Introduction to Queueing Theory. Macmillan, New York, 1972.

[4] Doshi, B. T., Queueing systems with vacations - A survey, Queueing Systems, Vol. 1, pp. 29-66, 1986.

[5] Franken, P., König, D., Arndt, U., and Schmidt, V., Queues and Point Processes, Wiley Series in Probability and Mathematical Statistics, 1982.

[6] Fuhrmann, S. W., and Cooper, R. B., Stochastic decompositions in the $M / G / 1$ queue with generalized vacations, Oper. Res. Vol. 33, No. 5, pp. 1117-1129, 1986.

[7] Fuhrmann, S. W., Inequalities for cyclic service systems with limited service disciplines, Proc. GLOBECOM '87, Tokyo, pp. 6.1.1-6.1.5, 1987.

[8] Lee, T. T., $M / G / 1 / N$ queue with vacation time and limited service discipline, Performance Evaluation, Vol. 9, pp. 181-190, 1989.

[9] Nain, P., Interdeparture times from a queueing system with preemptive resume priority, Performance Evaluation 4, pp. 93-98, 1984.

[10] Servi, L. D., Average delay approximation of $M / G / 1$ cyclic service queues with Bernoulli schedules. IEEE Journal on Selected Areas in Communications, Vol. SAC-4, No. 6, pp. 813-822, 1986. Correction in Vol. SAC-5, No. 3, p. 547, 1987.

[11] Stanford, D. A., Interdeparture-time distributions in the non-preemptive priority $\sum M_{i} / G_{i} / 1$ queue, Performance Evaluation 12, pp. 43-60, 1991.

[12] Stoyan, D., Comparison Methods for Queues and Other Stochastic Models, Wiley Series in Probability and Mathematical Statistics, 1983.

[13] Takagi, H., Queueing Analysis, Volume 1, North-Holland, 1991.

[14] Tedijanto, Stochastic comparisons in vacation models, Stochastic Models, Vol. 7, No. 1, pp. 125-135, 1991.

Shinsuke SHIMOGAWA

Optical \& Electronic Device department

ATR Optical and Radio Communications Research Laboratories

2-2 Hikaridai Seika-cho Soraku-gun, Kyoto 619-02, Japan

and

Yoshitaka TAKAHASHI

Performance Evaluation Research Group

NTT Telecommunication Networks Laboratories

9-11 Midori-cho, 3-Chome, Musachino-shi, 180, Japan 